\title{
The effect of scleral search coil lens wear on the
}

\section{eye}

\author{
Paul J Murphy, Anne L Duncan, Alastair J Glennie, Paul C Knox
}

\begin{abstract}
Backgroundlaim-Scleral search coils are used to measure eye movements. A recent abstract suggests that the coil can affect the eye by decreasing visual acuity, increasing intraocular pressure, and damaging the corneal and conjunctival surface. Such findings, if repeated in all subjects, would cast doubt on the credibility of the search coil as a reliable investigative technique. The aim of this study was to reassess the effect of the scleral search coil on visual function.

Methods-Six volunteer subjects were selected to undergo coil wear and baseline measurements were taken of $\log$ MAR visual acuity, non-contact tonometry, keratometry, and slit lamp examination. Four drops of $0.4 \%$ benoxinate hydrochloride were instilled before insertion of the lens by an experienced clinician. The lens then remained on the eye for $\mathbf{3 0}$ minutes. Measurements of the four ocular health parameters were repeated after 15 and 30 minutes of lens wear. The lens was then removed and the health of the eye reassessed.

Results-No obvious pattern of change was found in $\log$ MAR visual acuity, keratometry, or intraocular pressure. The lens did produce changes to the conjunctival and corneal surfaces, but this was not considered clinically significant.

Conclusion-Search coils do not appear to cause any significant effects on visual function. However, thorough prescreening of subjects and post-wear checks should be carried out on all coil wearers to ensure no adverse effects have been caused.

(Br f Ophthalmol 2001;85:332-335)
\end{abstract}

Sciences, Glasgow

Caledonian University, Glasgow, UK

P J Murphy

A L Duncan

A J Glennie

Department of Orthoptics, University of Liverpool,

Liverpool, UK

P C Knox

Correspondence to: Dr Paul J Murphy, Department of Vision Sciences, Glasgow Caledonian University, Cowcaddens Road, Glasgow G4 0BA, UK

P.Murphy@gcal.ac.uk

Accepted for publication 18 October 2000

Accurate measurement of the movements of the eyes is often required not only in studies of oculomotor function, but also in studies of the visual and vestibular systems. Accurate measurement of oculomotor parameters has been suggested to have a clinical role in the diagnosis and monitoring of disease. ${ }^{12}$ Various methods have been developed to record movements of the eyes including electrooculography, infrared oculography, video oculography, and scleral search coils. ${ }^{3}$ Although the most invasive of the available techniques, the scleral search coil method has gained wide acceptance because it provides excellent spatial and temporal resolution. ${ }^{4}$ It has been widely used in both animal and human research studies, in investigations of saccades, smooth pursuit, the vestibulo-ocular reflex, and optokinetic responses, in both normal and clinical subjects. $^{56}$

Briefly, the technique involves applying a coil of wire to the eye. In the case of human subjects, a contact lens or scleral annulus, in which the coil is embedded, is acutely placed in a locally anaesthetised eye for the length of an experiment, typically no longer than $45 \mathrm{~min}-$ utes. In animal subjects, coils can be implanted surgically. Movements of this coil in a magnetic field, generated by a set of field coils, induces an electric current in the eye coil proportional to its position in the field. This current, suitably amplified and filtered, provides, strictly speaking, an eye in space position signal. However, where the head does not move relative to the field coils, this is in effect converted to an eye in orbit position signal.

Given the wide use of this technique, the brief report published by Zacher et al claiming that standard coils could have serious effects on the ocular surface, intraocular pressure (IOP), and visual acuity (VA) caused concern. While obviously raising concerns over the ethics of the technique, such findings, if substantiated, would have implications for a large body of oculomotor results obtained using this technique. It was claimed that the acuity of their subjects declined by up to six lines during coil wear. As typically visual targets used in oculomotor studies are fractions of a degree in size, large changes in visual acuity would seriously modify subject performance.

We therefore decided to explore these issues further. Given the obvious ethical difficulties of embarking on essentially a safety study, we used a small group of carefully screened subjects, and limited coil exposure to only 30 minutes, well within the recommended period of 45 minutes.

\section{Materials and methods}

SUBJECTS

With local ethical approval, six subjects were recruited from the student population of Glasgow Caledonian University (three males, three females, mean age 22.17 years). All were screened before inclusion to ensure that there was no underlying ocular or systemic disease, and each gave informed written consent. None of the subjects were habitual contact lens wearers. Baseline measurements were recorded for the four study ocular parameters-visual acuity (VA), intraocular pressure (IOP), keratometry (K), and slit lamp examination (SL). Four drops of $0.4 \%$ benoxinate hydrochloride (Minims, Chauvin Pharmaceuticals) were instilled in each subject's right eye, and a 


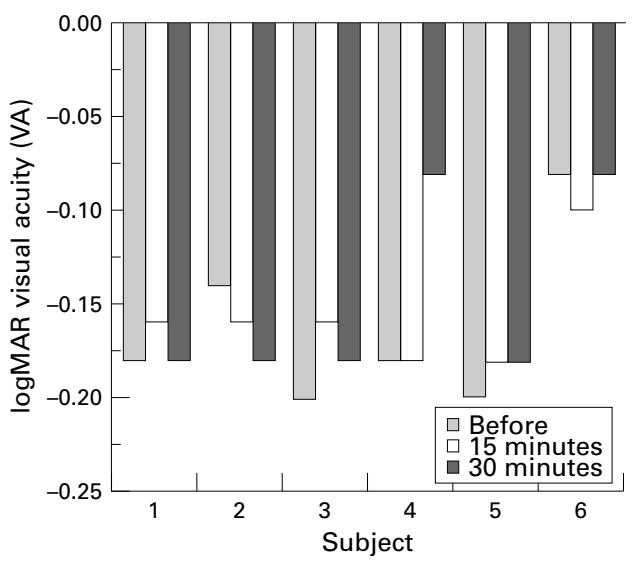

Figure $1 \log M A R$ visual acuities (VA) before lens insertion, and at 15 and 30 minutes after lens insertion, for each subject.

qualified optometrist (PJM) then inserted the scleral search coil annulus (Skalar Medical BV, Delft, Netherlands) in the right eye. After insertion, the subjects were encouraged to blink and move their eyes as normal, and the measurements for the four ocular health parameters were repeated after 15 and 30 minutes. The annulus was then removed, and the health of the eye reassessed.

\section{CORNEAL PARAMETERS ASSESSED}

Visual acuity (VA)

Visual acuity (that is, corrected vision) was assessed using a Bailey-Lovie logMAR chart. This chart is an improvement over the standard Snellen acuity chart since it provides a regular progression in letter size, with equal numbers of letters per line. Visual acuity is recorded in log units, with a progression of 0.1 log units from one line to the next. For this study, visual acuity was recorded both aided and unaided, and before annulus insertion, after 15 minutes of wear, after 30 minutes of wear, and after removal.

Intraocular pressure (IOP)

Intraocular pressure was measured using the Topcon CT-60 non-contact tonometer. This instrument uses a pulse of air to applanate the cornea, from which the instrument calculates the IOP at the moment the cornea is flattened. Goldmann tonometry, which is reported to be more accurate, could not be used since it can mark the anterior corneal surface, and we wished to observe the effects of scleral coil lens wear alone. Assessment of IOP was carried out twice only-before annulus insertion and after it was removed. An average of three measurements was taken to obtain a valid measure.

\section{Keratometry (K)}

Keratometry was measured, using a JavalSchiotz keratometer, to assess any large changes in corneal curvature produced by lens wear. Measurements were taken before annulus insertion and after removal.

Slit-lamp examination (SL)

A check of corneal health was performed before annulus insertion, and after removal, to assess any changes produced by the use of the

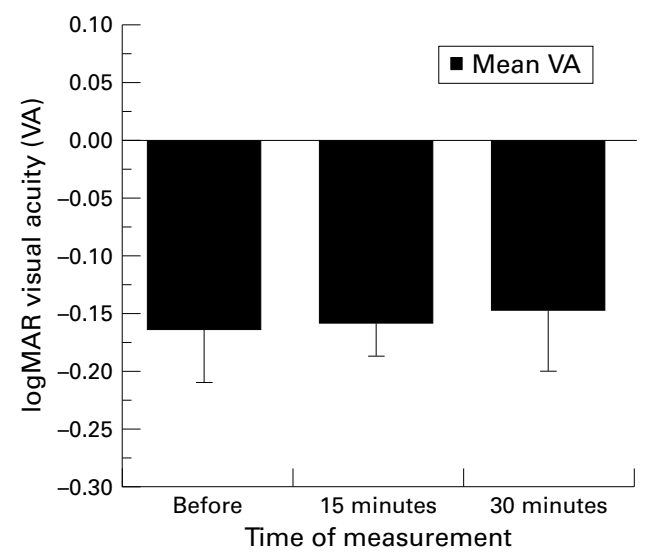

Figure 2 Mean $\log M A R$ visual acuities (VA) before lens insertion, and at 15 and 30 minutes after lens insertion.

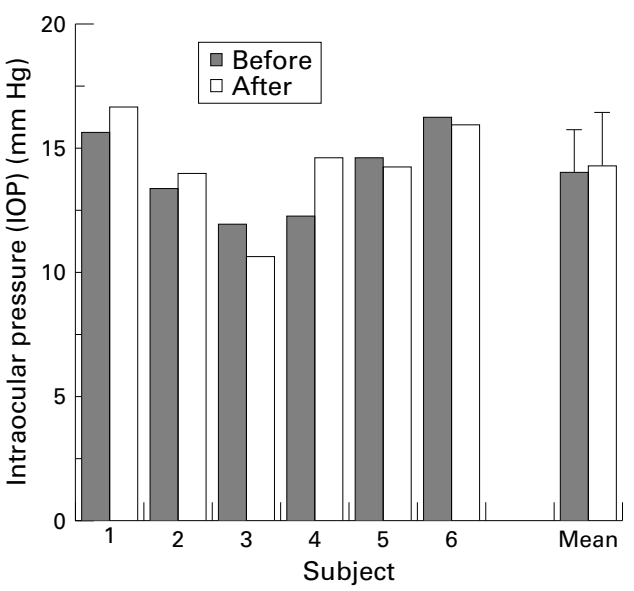

Figure 3 Individual and mean intraocular pressure (IOP) measurements before and after lens wear.

lens. The examination included observation of the lids and lashes, bulbar and palpebral conjunctiva, corneal clarity, and staining with sodium fluorescein. Conjunctival redness (bulbar, limbal, and palpebral) and corneal staining were assessed using the Cornea and Contact Lens Research Unit (CCLRU, University of New South Wales) grading scales. ${ }^{89}$

\section{Results}

VISUAL ACUITy (VA)

Visual acuities before lens insertion, and at 15 and 30 minutes after insertion, are plotted for each subject in Figure 1, and pooled mean data for all subjects are shown in Figure 2. There appeared to be small changes in acuity, but the pattern was mixed. Thus, subject 2 showed an improvement in VA of 0.04 units, while subject 4 showed a reduction in VA of 0.1 units, over the 30 minute wear period. However, there was no statistically significant change in visual acuity over the time period analysed (one way ANOVA, $\mathrm{p}=0.803$ ).

INTRAOCULAR PRESSURE (IOP)

The individual IOP measurements, and the mean IOP for all subjects, before and after lens wear are shown in Figure 3. There was no clear pattern of effect in the individual results, and while the mean result suggested a slight 


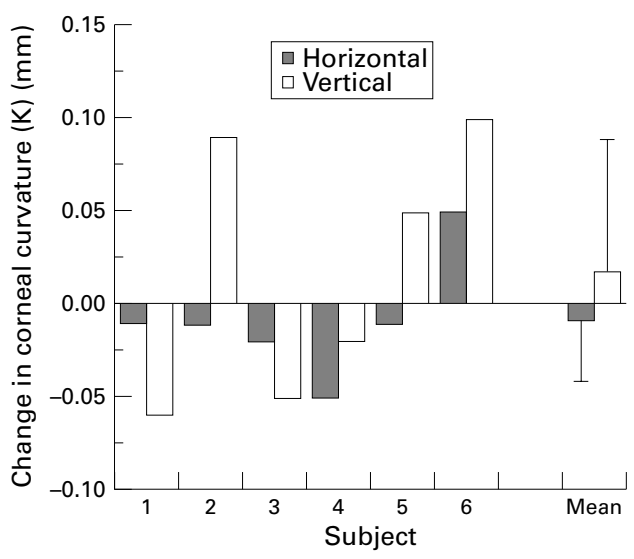

Figure 4 Individual and mean changes in central corneal curvature $(K)$ produced by scleral lens wear.

Table 1 Change in the grade of conjunctival redness, for each subject, after scleral lens wear

\begin{tabular}{llll}
\hline & \multicolumn{2}{l}{ Change in grade of conjunctival redness after coil wear } \\
\cline { 2 - 4 } Subject & Bulbar redness & Limbal redness & Palpebral redness \\
\hline 1 & +1.5 & +2.5 & 0 \\
2 & +2 & +1.5 & +1 \\
3 & +1 & +1 & 0 \\
4 & +1 & +1 & 0 \\
5 & +2 & +0.5 & 0 \\
6 & +1 & +1.5 & 0 \\
\hline
\end{tabular}

Table 2 Corneal staining grade, for each subject, after scleral lens wear

\begin{tabular}{lllll}
\hline Subject & Type & Extent & Depth & Area \\
\hline 1 & 0 & 0 & 0 & - \\
2 & 1 & 1 & 1 & 5 \\
3 & 0 & 0 & 0 & - \\
4 & 1 & 1 & 1 & $1,4,5$ \\
5 & 1 & 1 & 1 & 5 \\
6 & 1 & 1 & 1 & 5 \\
\hline
\end{tabular}

increase in IOP with lens wear, this was statistically insignificant ( $t$ test: before/after, $\mathrm{p}=0.774)$.

KERATOMETRY (K)

We also examined changes produced in central corneal curvature following lens wear (Fig 4). The size of change produced was typically less than $0.05 \mathrm{~mm}$, although in one case it changed by as much as $0.1 \mathrm{~mm}$. There was no consistent pattern across all the subjects, but there was a slight suggestion of larger effects in the vertical meridian, compared with horizontal. This may be due to the action of the lids, when blinking, pressing down on the scleral lens in this direction. The small changes in corneal curvature, observed in the horizontal and vertical meridians, were statistically insignificant ( $t$ test: horizontal, before/after $\mathrm{p}=0.95$; vertical, before/after $\mathrm{p}=0.90$ ).

SLIT LAMP EXAMINATION (SL)

The changes in grade of conjunctival redness, for each subject, are given in Table 1 , and the grading for corneal staining in Table 2. An example of the corneal staining found is given in Figure 5A, and Figure 5B shows the position of the scleral coil lens on the eye. For conjunctival redness, all of the subjects showed an increase in grade of bulbar and limbal redness.
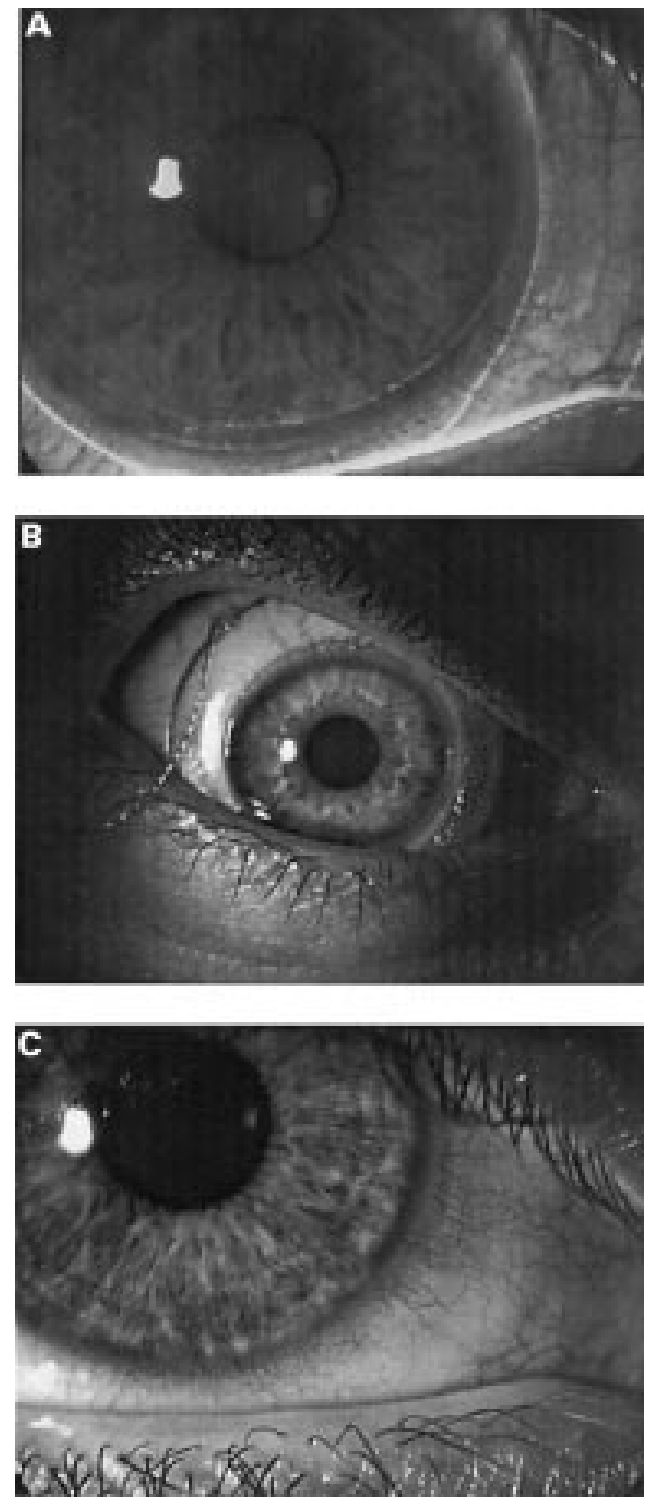

Figure 5 (A) Typical corneal staining produced by scleral lens wear. (B) Position of scleral search coil lens on the eye. (C) Typical limbal redness produced by scleral lens wear.

Only subject 2 showed an increase in palpebral redness. An example of the typical limbal redness observed is shown in Figure 5C. Four of the six subjects showed some evidence of corneal staining, whereas before lens wear, none of the subjects had staining. However, while staining was found, the extent and depth indicated only slight epithelial damage.

\section{Discussion}

As a method for accurately measuring the movements of the eyes, scleral search coils have won wide acceptance. Although invasive, requiring the insertion of a lens or annulus, they allow high spatial and temporal resolution measurements of horizontal, vertical, and torsional eye movements to be made. Many observations of importance for understanding the performance of the human oculomotor system have been made using this technique. Zacher et al claimed that a standard annulus, inserted and used in accordance with standard 
practice, produced a number of notable ocular and visual effects. If replicated, these findings would have serious implications for a large body of work. Among other things, they reported significant increases in IOP and large decreases in visual acuity. While some of their observations raise issues concerning the safety and ethics of the method, this last aspect, the reported decrease in visual acuity, raises a different type of concern. Scleral search coils have been used in a large number studies in which subjects respond to relatively small visual targets of a few tenths of a degree. Large decreases in acuity due to the measurement technique would cause large artefactual alterations in oculomotor performance. This would be a serious confound in this type of study. Hence the importance of trying to replicate the findings of Zacher et al. ${ }^{7}$

We clearly failed to replicate the findings of Zacher et al. Apart from some conjunctival redness and corneal staining, which might be expected, the insertion of coils produced no clinically significant ocular changes. Furthermore, the conjunctival and corneal effects were only of a superficial, transient, nature. Importantly, we found no significant effects of coil wear on visual acuity. Thus our results confirm that scleral search coil wear has no effect on the ability of subjects to perform oculomotor tasks.

Zacher et al reported irritation of the eyelids, bulbar conjunctival redness, a $5 \mathrm{~mm} \mathrm{Hg}$ increase in IOP, grade 2 and 3 corneal staining, a "five line decrease in Snellen acuity", and a buckled iris. However, there is no indication as to whether these effects were found in one patient or in a number of different patients. The irritation of the eyelids and bulbar redness we have observed, but the change in IOP, corneal staining, VA, and the buckled iris we have not. The effect of IOP change and iris buckling suggest that the annulus may have squeezed the anterior chamber angle, blocking aqueous outflow and distorting the ciliary body and iris root. The change in corneal staining and VA suggest a drying of the anterior corneal surface, as a result of insufficient or incomplete blinking patterns, where the annulus is interfering with lid movement. Our subjects were asked to blink and move their eyes as normal, and this may have prevented some effects being manifest. Differences in corneal exposure changes could also be exacerbated by the annulus being on the eye for 45 minutes, while we limited exposure to 30 minutes. This was chosen since 30 minutes more closely approximates a typical wearing time period during an eye movement experiment, and because the effect of $0.4 \%$ benoxinate begins to wear off at this time.

In placing the annulus in the eye, two methods are available. A metal applicator is available to aid placement, or it can be placed by hand. The first method could lead to excessive pressure being employed, forcing the lens against the eye. This might explain some of the changes found by Zacher et al. In our study, the annulus was inserted into the eye by hand, without the aid of the applicator, by an experienced contact lens clinician. This avoided the need for any unnecessary additional manipulation.

The young mean age of our subjects $(22.17$ years) should not influence the outcome of this study. The effect of increasing age on corneal fragility does not become a significant factor until after the age of 60 years, ${ }^{10}$ and the vast majority of subjects who require, or volunteer for, this procedure will be below this age.

In conclusion, the scleral search coil lens can produce significant effects in some subjects, especially if the lens is applied to the eye in an aggressive manner, or if the subject is not encouraged to blink frequently to re-wet the anterior corneal surface. We suggest that subjects who are selected for scleral search coil lens wear should be prescreened before wear to assess their baseline ocular health, and where possible, be excluded if corneal or ocular disease is present. Subjects should also be assessed post-lens wear to ensure no significant damage has occurred, which may require prophylactic treatment, as necessary.

1 Flipse JP, Straatof CSM, van der Steen JV, et al. Binocular accadic eye movements in multiple sclerosis. $\mathcal{F}$ Neurol Sci 1997;148:53-65.

2 Barton J S. Quantitative ocular tests for myasthenia gravis: a comparative review with detection theory analysis. $\mathcal{F}$ Neurol Sci 1998;155:104-14.

3 Carpenter RHS. Movement of the eyes. 2nd ed. London: Pion, 1988.

4 Collewijn H, van der Mark F, Jansen TC. Precise recording of human eye movements. Vis Res 1975;14:447-50.

5 Carl J, Gellman R. Human smooth pursuit: stimulusdependent responses. $\mathcal{F}$ Neurophysiol 1987;57:1446-63.

6 Collewijn H, Erkelens CJ, Steinman RM. Binocular co-ordination of human horizontal saccadic eye movements. F Physiol 1988;404:157-82.

7 Zacher JE, Irving EL, Allison RA, et al. The effect of scleral coil wear on visual function and acuity. Invest Ophthalmol Vis Sci 1998;39:4:S767.

8 Woods R. Quantitative slit lamp observations in contact lens practice. F Br Contact Lens Assoc (Scientific Meetings) 1989; $12: 42-5$.

9 Cornea and Contact Lens Research Unit. CCLRU Grading Scales. Sydney, Australia: School of Optometry, University of New South Wales (available from Vistakon), 1996.

10 Millodot M, Owens $\mathrm{H}$. The influence of age on the fragility of the cornea. Acta Ophthalmol 1984;62:819-24. 